\title{
CARACTERÍ́STICAS DE LA ENFERMEDAD PERIODONTAL EN PACIENTES ANÉMICOS Y NO ANÉMICOS
}

\author{
FEATURES OF PERIODONTAL DISEASE IN ANEMIC AND NON-ANEMIC PATIENTS \\ CARACTERISTICAS DA DOENCA PERIODONTAL EM PACIENTES ANÊMICOS E NÃO ANÊMICOS
}

\author{
ARIANNA PAZ REESE', GEOCONDA LUZARDO JURADO' \\ 1 Universidad Católica de Santiago de Guayaquil, Guayaquil, Ecuador
}

\begin{abstract}
Resumen
Introducción: la anemia relacionada a enfermedades crónicas es el tipo más frecuente de anemias; se puede producir en presencia de procesos inflamatorios, neoplásicos o infecciones crónicas. La enfermedad periodontal es un proceso inflamatorio del periodonto, que acorta la vida de los eritrocitos e interrumpe la eritropoyesis disminuyendo el número de eritrocitos, hemoglobina y hematocrito. Objetivo: determinar la prevalencia de enfermedad periodontal en pacientes anémicos y no anémicos. Hipótesis: la anemia exacerba la enfermedad periodontal. Métodos: se estudió una serie de casos clínicos compuesto por 100 pacientes con enfermedad periodontal entre 20 a 63 años atendidos en la Clínica odontológica-UCSG. Se dividió la muestra en 2 grupos; un grupo que consistió en 50 pacientes no anémicos con enfermedad periodontal; y el otro grupo con 50 pacientes anémicos con enfermedad periodontal. A los 2 grupos de pacientes se les realizó un examen de sangre con el fin de medir el número de eritrocitos, hemoglobina y hematocrito. Resultados: se observó que del $50 \%$ de anémicos, el $26 \%$ presentó gingivitis asociada a placa y el $24 \%$ periodontitis, siendo más predominante en un $9 \%$ la periodontitis crónica moderada. Conclusión: no se encontró grandes diferencias clínicas en los pacientes que padecieron la enfermedad periodontal con anemia y sin anemia. Se recomienda realizar estudios con una muestra mayor con el fin de investigar la asociación entre la enfermedad periodontal y/o anemia, así como el efecto del tratamiento periodontal en estos pacientes.
\end{abstract}

PALABRAS CLAVE: periodontitis anemia, eritrocitos, hemoglobina.

Abstract

Introduction: anemia related to chronic diseases is the most frequent type of anemia, it can occur in the presence of inflammatory, neoplastic or chronic infections. Periodontal disease is an inflammatory process of the periodontium, which shortens the life of erythrocytes and interrupts erythropoiesis thus decreasing the number of erythrocytes, hemoglobin and hematocrit. Objective: to determine the prevalence of periodontal disease in anemic and nonanemic patients. Hypothesis: anemia exacerbates periodontal disease. Methods: a series of clinical cases was studied, consisting of 100 patients with periodontal disease between 20 and 63 years of age treated at the Dental Clinic of UCSG. The sample was divided into 2 groups. A group consisting of 50 non-anemic patients with periodontal disease; and the other group of 50 anemic patients with periodontal disease. The two groups of patients underwent a blood test in order to measure the number of erythrocytes, hemoglobin and hematocrit. Results: We observed that from the $50 \%$ of anemic patients, $26 \%$ presented gingivitis associated with plaque and $24 \%$ periodontitis, being more prevalent in $9 \%$ the moderate chronic periodontitis. Conclusion: no large differences were found in the characteristics of periodontal disease among patients with anemia and without anemia. It is recommended to perform studies with a larger sample in order to investigate the association between periodontal disease and anemia. As well as the effect of periodontal treatment in these patients

KEYWORDS: periapical periodontitis, anemia, erythrocyte, hemoglobin.

\section{Resumo}

Introdução: a anemia relacionada as doenças crônicas é o tipo mais frequente de anemia, podendo ocorrer na presencia de processos inflamatório, neoplasicos o infecções crônicas. A doença periodontal é um processo inflamatório do periodonto, que encurta a vida dos eritrócitos e interrompe a eritropoiese ao diminuir o número de eritrócitos, hemoglobina e hematócrito. Objetivo: determinar na prevalência de doença periodontal em pacientes anêmicos e não anêmicos. Hipótese: a anemia exacerba a doença periodontal. Métodos: uma serie de casos clínicos foi estudada, consistindo de 100 pacientes com doença periodontal entre 20 e 63 anos de idade, atendidos na Clínica Odontológica - UCSG. A mostra foi dividida em dois grupos. Um grupo foi conformado de 50 pacientes no anêmicos com doença periodontal; e outro de 50 pacientes anêmicos com doença periodontal. Os dois grupos de pacientes foram sometidos a um exame de sangre com o objetivo de medir e número de eritrócitos, hemoglobina e hematócrito. Resultados: observou-se que $50 \%$ dos pacientes anêmicos, $26 \%$ apresentavam gengivite associada à placa e $24 \%$ periodontite, sendo mais prevalente em periodontite crônica moderada em 9\%. Conclusões: não foram encontradas grandes diferenças nas características de pacientes com doença periodontal entre os pacientes com anêmica e sem anemia. Recomendasse a realização de estudos com uma amostra maior com o fim de investigar a associação entre a doença periodontal e anemia. Assim como 0 efeito de tratamento periodontal nesses pacientes.

PALABRAS-CHAVE: periodontite periapical, anemia, aritrócitos, hemoglobina. 
INTRODUCCIÓN

La enfermedad periodontal es un proceso inflamatorio del periodonto de protección e inserción, producida por cerca de 400 patógenos específicos que provocan tanto una destrucción a nivel del ligamento periodontal como del hueso alveolar, y en consecuencia produce una bolsa periodontal o recesión de la encía..$^{1-2}$ Las bacterias gramnegativas y sus productos, como los lipopolisacáridos acceden por el epitelio del surco, el cual se encuentra inflamado y ulcerado, e ingresan a la circulación..$^{3-7}$ En efecto, crean una leve inflamación sistémica, que se manifiesta en los cambios de los componentes de la sangre..$^{8-12}$ La respuesta inflamatoria de la enfermedad periodontal permite el paso a más bacterias y productos, enzimas hidrolíticas y peptidoglicanos que entran a la circulación. En respuesta, el huésped produce citoquinas y mediadores como interleuquinas y prostaglandinas., ${ }^{2,13-15}$

A la enfermedad periodontal se la considera una infección crónica que promueve el secuestro de hierro e inhibe la producción y la sobrevivencia de los glóbulos rojos. Por consiguiente, la eritropoyesis es defectuosa provocando bajos niveles de hemoglobina, hematócrito y número de eritrocitos. ${ }^{4,16-20}$

La periodontitis debería ser considerada un factor de riesgo de la anemia ya que el tratamiento de la misma podrá restablecer el número de glóbulos rojos y hemoglobina..$^{21-24}$ Con el presente estudio se desea conocer si existen diferencias en las características de la enfermedad periodontal entre los pacientes con y sin anemia

\section{MATERIALES Y MÉTODOS}

Se realizó un estudio transversal donde se incluyeron 100 pacientes con enfermedad periodontal, entre 20 y 63 años atendidos en la clínica odontológica de la Universidad Católica Santiago de Guayaquil (UCSG), de Ecuador, en el periodo comprendido entre el 14 de octubre del 2013 al 30 de enero del 2014.

Los criterios de inclusión fueron pacientes de 20 a 63 años, con diagnóstico de periodontitis o gingivitis que asistieran a la clínica odontológica UCSG en el periodo previamente especificado. Se excluyeron pacientes que donaran sangre en los últimos 6 meses, mujeres embarazadas o en periodo de lactancia, pacientes fumadores y diabéticos.
Para el estudio se solicitó a todos los pacientes firmar el consentimiento informado, se les realizó un periodontograma, que incluía nivel de margen gingival $(\mathrm{MC})$, profundidad de sondaje (PS) y nivel de inserción clínica (NIC), con el fin de establecer un diagnóstico periodontal para cada paciente.

Posteriormente a los 100 pacientes se les realizó un examen de sangre con el fin de medir el número de eritrocitos, hemoglobina y hematocrito, dividiendo la muestra en 2 grupos. El primero integrado por 50 pacientes no anémicos con enfermedad periodontal y el segundo grupo, con 50 pacientes anémicos con enfermedad periodontal.

La muestra fue documentada y tabulada, con las respectivas pruebas estadísticas, mediante el uso del software Microsoft Excel versión 14.0 ${ }^{\circledR}$. El presente estudio siguió las recomendaciones de la Declaración de Helsinki de 2013, cumpliendo con los principios éticos para la investigación médica en seres humanos.

\section{RESULTADOS}

De los 100 pacientes estudiados 64 eran de sexo femenino y 36 de sexo masculino. Al subdividirlos según la presencia de anemia, se observó que 38 pacientes de sexo femenino la presentaban, siendo el subgrupo de mayor cantidad de pacientes. Tabla 1.

TABLA 1. DISTRIBUCIÓN PORCENTUAL DE ANEMIA SEGÚN EL GÉNERO

\begin{tabular}{lcrr}
$\begin{array}{l}\text { PRESENCIA DE } \\
\text { ANEMIA }\end{array}$ & \multicolumn{2}{c}{ GÉNERO } & $\begin{array}{r}\text { TOTALES } \\
\text { PRESENCIA DE } \\
\text { ANEMIA }\end{array}$ \\
\cline { 2 - 4 } $\mathrm{Si}$ & $12 \%$ & $38 \%$ & $50 \%$ \\
\hline No & $24 \%$ & $26 \%$ & $50 \%$ \\
\hline Totales género & $36 \%$ & $64 \%$ & $100 \%$
\end{tabular}

Al subdividirlos por edad se encontró que el subgrupo de mayor frecuencia fueron 19 pacientes anémicos que tenían de 20 a 30 años. Tabla 2.

TABLA 2. DISTRIBUCIÓN DE PACIENTES QUE PRESENTAN ANEMIA POR EDAD

\begin{tabular}{|c|c|c|c|c|c|}
\hline \multirow{2}{*}{$\begin{array}{l}\text { PRESENCIA DE } \\
\text { ANEMIA }\end{array}$} & \multicolumn{4}{|c|}{ EDAD } & \multirow{2}{*}{$\begin{array}{r}\text { TOTALES PRESNCI } \\
\text { DE ANEMIL }\end{array}$} \\
\hline & $20-30$ & $31-41$ & $42-52$ & $53-63$ & \\
\hline $\mathrm{Si}$ & $19 \%$ & $14 \%$ & $11 \%$ & $6 \%$ & $50 \%$ \\
\hline No & $13 \%$ & $10 \%$ & $12 \%$ & $15 \%$ & $50 \%$ \\
\hline Totales género & $32 \%$ & $24 \%$ & $23 \%$ & $21 \%$ & $100 \%$ \\
\hline
\end{tabular}


En la tabla 3 se describe la enfermedad periodontal y la anemia. De los pacientes con anemia, 26 presentaban gingivitis asociada a placa. Además nueve pacientes padecían periodontitis crónica localizada moderada o periodontitis crónica generalizada severa.

TABLA 3. DISTRIBUCIÓN PORCENTUAL DE ENFERMEDAD PERIODONTAL EN ANEMIA

\begin{tabular}{lrrr} 
& PRESENCIA & TOTALES \\
ENFERMEDAD PERIODONTAL & DE ANEMIA & ENFERMEDAD \\
& SI & NO & PERIODONTAL \\
\hline Gingivitis asociada a placa & $26 \%$ & $28 \%$ & $54 \%$ \\
\hline Periodontitis crónica generalizada leve & $0 \%$ & $0 \%$ & $0 \%$ \\
\hline Periodontitis crónica generalizada severa & $9 \%$ & $11 \%$ & $20 \%$ \\
\hline Periodontitis crónica generalizada moderada & $1 \%$ & $4 \%$ & $5 \%$ \\
\hline Periodontitis crónica localizada leve & $0 \%$ & $0 \%$ & $0 \%$ \\
\hline Periodontitis crónica localizada severa & $5 \%$ & $5 \%$ & $10 \%$ \\
\hline Periodontitis crónica localizada moderada & $9 \%$ & $2 \%$ & $11 \%$ \\
\hline Totales presencia de anemia & $50 \%$ & $50 \%$ & $100 \%$
\end{tabular}

En la tabla 4 se relaciona la enfermedad periodontal y el nivel de hemoglobina. Se evidenció, que existe un $54 \%$ de pacientes con niveles normales de hemoglobina que padecen gingivitis asociada a placa, de los cuales el $10 \%$ de pacientes son de género masculino y $18 \%$ femenino. En relación al $11 \%$ que padecía periodontitis crónica localizada moderada, el 3\% fueron de género masculino y $6 \%$ femenino, presentaron un nivel de hemoglobina bajo.

\section{TABLA 4. DISTRIBUCIÓN DE PACIENTES QUE PRESENTAN ENFERMEDAD PERIODONTAL POR NIVEL DE HEMOGLOBINA}

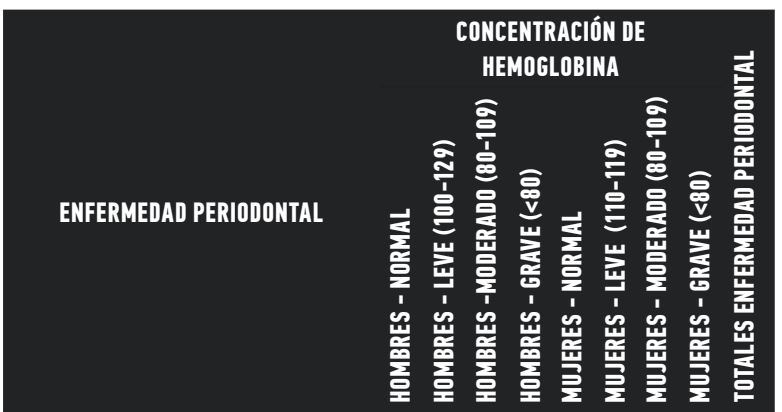

Gingivitis asociada a placa $\begin{array}{llllllll}10 \% & 4 \% & 0 \% & 0 \% & 18 \% & 20 \% & 2 \% & 0 \%\end{array}$

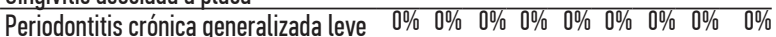
\begin{tabular}{lllllllll}
\hline Periodontitis crónica generalizada & $9 \%$ & $2 \%$ & $0 \%$ & $0 \%$ & $2 \%$ & $7 \%$ & $0 \%$ & $0 \%$
\end{tabular} severa

\begin{tabular}{llllllllll}
\hline Periodontitis crónica generalizada & $2 \%$ & $0 \%$ & $0 \%$ & $1 \%$ & $2 \%$ & $0 \%$ & $0 \%$ & $0 \%$ & $5 \%$
\end{tabular} moderada

\begin{tabular}{llllllllll}
\hline Periodontitis crónica localizada leve & $0 \%$ & $0 \%$ & $0 \%$ & $0 \%$ & $0 \%$ & $0 \%$ & $0 \%$ & $0 \%$ & $0 \%$
\end{tabular}

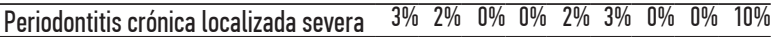
Periodontitis crónica localizada $\begin{array}{llllllllllll}0 \% & 3 \% & 0 \% & 0 \% & 2 \% & 6 \% & 0 \% & 0 \% & 11 \%\end{array}$ moderada

Totales concentración de hemoglobina $24 \% 11 \% \quad 0 \% \quad 1 \% 26 \% 36 \% \quad 2 \% \quad 0 \% 100 \%$

En la tabla 5 se relaciona la enfermedad periodontal y el nivel de hematocrito. Se vereficó que existe un $54 \%$ de pacientes con niveles normales de hematocrito que padecen gingivitis asociada a placa, de los cuales el $7 \%$ de pacientes son de género masculino y 23\% femenino. En relación al $11 \%$ que padecía periodontitis crónica localizada moderada, el $3 \%$ de género masculino y $4 \%$ femenino presentaron una concentración de hematocrito bajo.

TABLA 5. DISTRIBUCIÓN PORCENTUAL DE ENFERMEDAD PERIODONTAL EN RELACIÓN AL NIVEL DE HEMATOCRITO

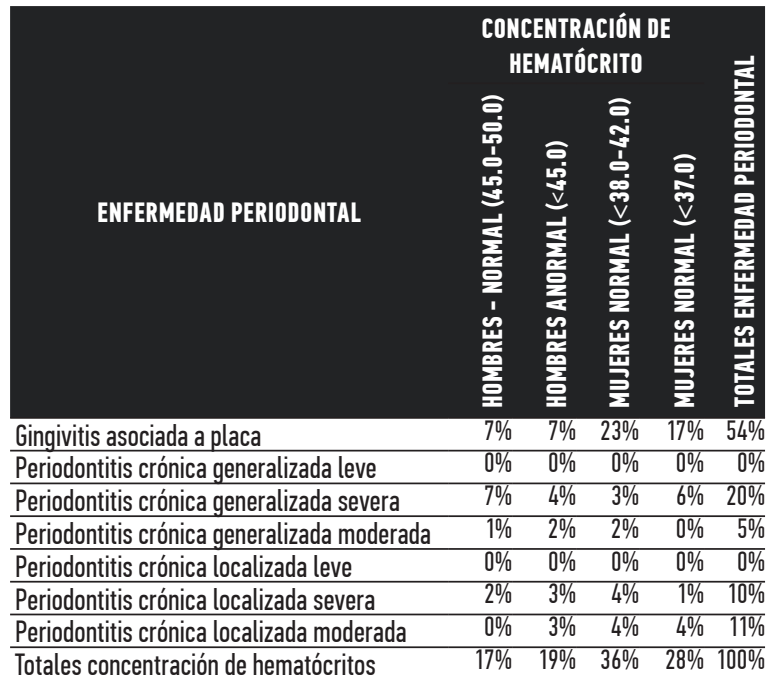

En la tabla 6 se apreció que existe un número mayor de pacientes con gingivitis asociada a placa, $54 \%$; de los cuales el $13 \%$ de pacientes, masculino y $25 \%$ femenino que presentaron un recuento eritrocitario normal. Analizando la muestra completa la mayoría de pacientes presentó un recuento normal de eritrocitos.

TABLA 6. DISTRIBUCIÓN PORCENTUAL DE ENFERMEDAD PERIODONTAL EN RELACIÓN AL RECUENTO DE ERITROCITOS

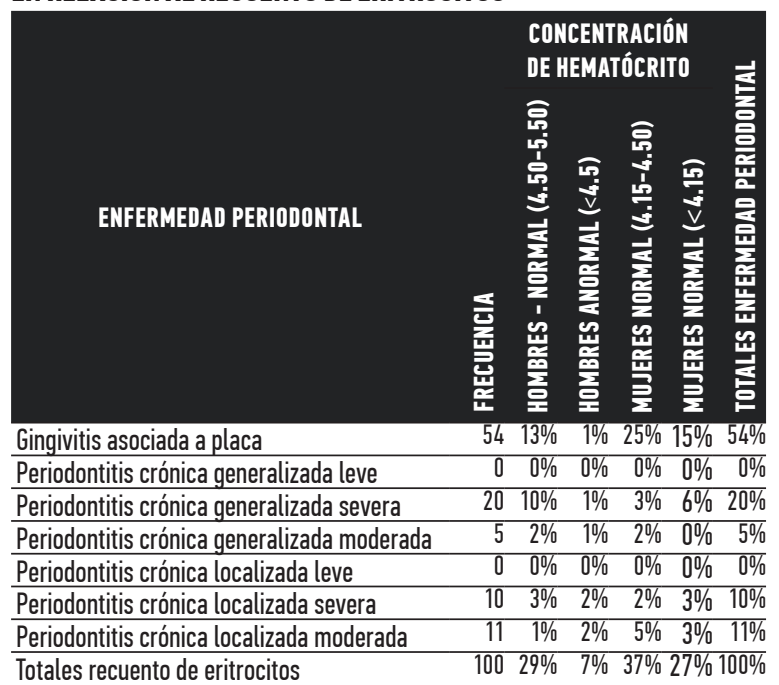

DISCUSIÓN

Varios autores han reportado que la periodontitis crónica produce anemia y proveen evidencia que el tratamiento no quirúrgico periodontal 
incrementa los valores sanguíneos de elementos figurados (eritrocitos + hemoglobina) en dichos pacientes. ${ }^{1,13,24}$ Rodak B et al, ${ }^{25}$ establecieron en su estudio que el número de eritrocitos y hemoglobina son significativamente bajos en pacientes que padecen periodontitis en comparación a los sanos. Kolte et al. ${ }^{26}$ también establecen una relación positiva a la disminución del recuento eritrocitario y la progresión de la enfermedad periodontal. Anand $\mathrm{N}$ et al, ${ }^{17}$ relacionan la enfermedad periodontal a la anemia, estableciendo como causante a la hepcidina. Debido a que las citoquinas proinflamatorias que se producen en la periodontitis, estimulan a la hepcidina, que es la hormona que regula el hierro y la eritropoyesis. Una inflamación crónica produce anemia.

En el presente estudio, al comparar con el grupo control se estableció que la mayor parte de pacientes que padecen enfermedad periodontal presentan un recuento eritrocitario, nivel de hemoglobina y hematocrito normal, al igual que en los estudios de Aljohani H., Krishna P10 y Sánchez S. ${ }^{13}$ Sin embargo se observó que los pacientes que padecían periodontitis crónica localizada moderada presentaban una concentración baja tanto de hematocrito como de hemoglobina. Concuerda con el estudio de Malhotra R et al, ${ }^{4}$ el cual establece que la periodontitis crónica, por ser una enfermedad más de tipo crónica con respecto a la gingivitis, disminuyen los valores hematológicos

El presente estudio investigó las diferentes características de la enfermedad periodontal en los pacientes con y sin anemia.

Musalaiah et al1, Malhotra et al13, Dhananjay $\mathrm{M}$ et al, Agarwal $\mathrm{N}$ et al, ${ }^{24}$ reportaron que la periodontitis crónica produce anemia y proveen evidencia que el tratamiento no quirúrgico periodontal incrementa los valores sanguíneos en dichos pacientes.

CONCLUSIONES

La mayor parte de pacientes que padecen enfermedad periodontal presentan un recuento eritrocitario, nivel de hemoglobina y hematocrito normal. Sin embargo, en los que padecían periodontitis crónica localizada moderada fue más frecuente una concentración baja tanto de hematocrito como de hemoglobina. Debido al diseño epidemiológico del estudio y al tamaño de la muestra los resultados presentados no son concluyentes, por lo que se recomienda desarrollar estudios longitudinales con una muestra mayor, en donde se puedan ajustar estadísticamente las variables de edad y género, con el fin de determinar la relación entre las diferentes características de la enfermedad periodontal y la anemia.

\section{REFERENCIAS BIBLIOGRÁFICAS}

1. Musalaiah S, Anupama M, Nagasree M, Murali Ch, Aravind K, Mohan P. Evaluation of nonsurgical periodontal therapy in chronic periodontitis patients with anemia by estimating hematological parameters and high-sensitivity C-reactive protein levels. JPBS. 2014 Julio [accesado 2014 octubre 15] ; 6(1): 64-69.

2. Nubesh K, Safar I, Mohamed H. Sabari C, Kumar S. Relationship between Hemoglobin Level and Severity of Chronic Periodontitis. Int. J. Chem. \& LifeSci.2014[accesado 2014 octubre 15], 3 (01), 12691273

3. Pan-pan CH, Jiao X, Wang X, Lin J, Cai M. Hepcidin expression in anemia of chronic disease and concomitant iron-deficiency anemia. Clin Exp Med. 2011[accesado 2014 diciembre 10],11:33-42.

4. Sahgal A, Kharsan V, Makkad R, Agrawal A, Shailendra, Chaturvedi S, Tanwani T. To Compare the Erythrocytes Count and Level of Hemoglobin in Chronic Periodontitis Patients with Control Subjects. Journal of Dental Peers. 2013[accesado 2014 octubre 15]; 1(2) 76-82.

5. Hatipoglu H, Gungor M, Cagirankaya L, Caglayan F. Severe periodontal destruction in a patient with advanced anemia. A case report. EJD. 2012[accesado 2014 octubre 15]; 6: 95-100.

6. Prathap S, Hedge S, Rajesh K, Kumar M. Anemia-a risk factor for periodontitis. KDJ. 2010[accesado 2014 octubre 15]; 33(3):173-175.

7. Erdemir E, Nalcaci R, Caglayan O. Evaluation of Systemic Markers Related To Anemia of Chronic Disease in the Peripheral Blood of Smokers and Non-Smokers with Chronic Periodontitis.Eur J Dent. 2008 Abril [accesado 2014 diciembre 16]; 2: 102-109.

8. Vanzato C, Marçon E, Rico J, Corrêa B, Salimon F, Partata E. Influencia de la enfermedad periodontal sobre las células sanguíneas y los niveles de proteína c reactiva. AOV.2011[accesado 2014 octubre 15];4(2):19. Disponible en www.actaodontologica.com/ediciones/2011/2/art20.asp

9. Martínez Y, García I, Llamosas E. Estudio comparativo de diversos elementos sanguíneos en pacientes con y sin periodontitis. Revista Nacional de Odontología de Mexico.2013[accesado 2014 octubre 15]; 4. Disponible en: http://www.intramed.net/ contenidover.asp?contenidoID $=82980$

10. Krishna P, Vamshidhar R , Arjun S, Ratna V, Gowtham K, Dimple A. An Investigation of Blood Hemogram and Estimation of Serum Iron and Pro- 
tein Levels in Aggressive Periodontitis. Patients: A Clinic Biochemical Study. JCDP. 2013[accesado 2014 octubre 15]; 14(5): 852-857.

11. Oppermann V. An overview of the epidemiology of periodontal diseases in Latin America. BOR.2007 [accesado 2014 diciembre 26];21(1):8-15.

12. WHO Global Database on Anaemia[database on the Internet] España: Benoist B, McLean E, Egli I, Cogswell M. 2007. [accesado 2015 enero 20]. [about 51p.]. Disponible en: http://whqlibdoc.who.int/ publications/2008/9789241596657_eng.pdf

13. Malhotra R, Kapoor, Grover V, Grover D, Kaur A. Effect of scaling and root planning on eritrocyte count, hemoglobin and hematocrit in patients with chronic periodontal disease. JDH. 2012[accesado 2014 octubre 15]; 89(3): 195-203.

14. Eley B, Soory M, Manson J. Etiología de la enfermedad periodontal. Periodoncia. España: Elsevier editorial;2012. p36 - 38. [accesado 2014 Noviembre 6]

15. Patil S, Rao RS, Sanketh DS, Amrutha N. Microbial Flora in Oral Diseases. J Contemp Dent Pract.2013[accesado 2014 octubre 6];14(6):1202-1208.

16. Botero J, Bedoya. Determinantes del Diagnóstico Periodontal. Rev. Clin. Periodoncia Implantol. Rehabil. Oral.2010[accesado 2014 octubre 6]; 3(2): 94-99.

17. Sakalauskiene J, Giedrimiene D, Kubilius R, Gleiznys A, Vitkauskiene A, Ivanauskiene E, Puskunigyte K. Cytokine production by leukocytes in patients with periodontitis. Cent. Eur. J. Med. 2014[accesado 2015 enero 4]; 9(6):821-829

18. Artese L, Maciej J, Piattelli A, Ferrari D, Luciana A, Cardoso G, et al. Immunohistochemical analysis of inflammatory infiltrate in aggressive and chronic periodontitis: a comparative study. Clin Oral Invest. .2011[accesado 2015 enero 4]15:233-240.

19. Carranza F, Newman M, Takei H. Clasificación de enfermedades y lesiones que afectan el periodoncio. En: Novak M,editor. Periodontología clínica. México: McGraw Hill Interamericana;2004. [accesado 2014 diciembre 18]p.66-75.
20. Bullon P, Cordero M, Quiles J, Ramirez-Tortosa M, Gonzalez A, Alfonsi S, García-Marín R, et al. Autophagy in periodontitis patients and gingival fibroblasts: unraveling the link between chronic diseases and inflammation. BMC Medicine.2012[accesado 2014 diciembre 18], 10:122. Disponible en: http:// www.biomedcentral.com/1741-7015/10/122

21. Sánchez S, Bascones I, Martínez A. Otras enfermedades periodontales I:Periodontitis como manifestaciones de enfermedades sistémicas. Avances de periodoncia.2008[accesado 2014 octubre 15]; 20(1):59-66.

22. Sigismond L, Baron G, Driss F, Westerman M, Herve P, Boutron I. Carole Beaumont,et al. Diagnostic accuracy of serum hepcidin for iron deficiency in critically ill patients with anemia. Intensive Care Med.2010[accesado 2014 octubre 15];36:1044-1048.

23. Echeverría J, Echeverría A, Santamaria M. Diagnóstico y sondaje periodontales. En: Echeverría J, editor. Peridoncia, cirugía periodontal e implantología. Barcelona:Ergon;2011[accesado 2014 diciembre 18] p.39-44.

24. Agarwall N, Kumar V, Gujjari S. Effect of periodontal therapy on hemoglobin and erytrocyte levels in chronic generalized periodontitis patients: An interventional study. JISP. 2009[accesado 2014 octubre 15]; 13 (1):6-11.

25. Rodak B. Anemias: morfología de los eritrocitos y enfoque diagnóstico.En:Bell A,editor.Hematología Fundamentos y aplicaciones clínicas. Buenos Aires:Editorial médica Panamericana;2010. 202-210.

26. Kolte R, Kolte A, Deshpande N. Assessment and comparison of anemia of chronic disease in healthy subjects and chronic periodontitis patients: A clinical and hematological study. JISP.2014[accesado 2014 octubre 15]; 18(2):183-186. 